ARTICLE

DOI: $10.1038 / s 41467-017-01877-7$

\title{
NSD1- and NSD2-damaging mutations define a subset of laryngeal tumors with favorable prognosis
}

Suraj Peri ${ }^{1}$, Evgeny Izumchenko², Adrian D. Schubert (10 2,6, Michael J. Slifker ${ }^{1}$, Karen Ruth¹, Ilya G. Serebriiskii ${ }^{3}$, Theresa Guo ${ }^{2,4}$, Barbara A. Burtness ${ }^{5}$, Ranee Mehra 2,4 , Eric A. Ross ${ }^{1}$, David Sidransky ${ }^{2,4}$ \& Erica A. Golemis (1) ${ }^{3}$

Squamous cell carcinomas of the head and neck (SCCHN) affect anatomical sites including the oral cavity, nasal cavity, pharynx, and larynx. Laryngeal cancers are characterized by high recurrence and poor overall survival, and currently lack robust molecular prognostic biomarkers for treatment stratification. Using an algorithm for integrative clustering that simultaneously assesses gene expression, somatic mutation, copy number variation, and methylation, we for the first time identify laryngeal cancer subtypes with distinct prognostic outcomes, and differing from the non-prognostic laryngeal subclasses reported by The Cancer Genome Atlas (TCGA). Although most common laryngeal gene mutations are found in both subclasses, better prognosis is strongly associated with damaging mutations of the methyltransferases NSD1 and NSD2, with findings confirmed in an independent validation cohort consisting of 63 laryngeal cancer patients. Intriguingly, NSD1/2 mutations are not prognostic for nonlaryngeal SCCHN. These results provide an immediately useful clinical metric for patient stratification and prognostication.

\footnotetext{
${ }^{1}$ Biostatistics and Bioinformatics Division, Fox Chase Cancer Center, Philadelphia, 19111 PA, USA. ${ }^{2}$ Department of Otolaryngology-Head and Neck Surgery, Johns Hopkins University School of Medicine, Baltimore, MD 21287, USA. ${ }^{3}$ Molecular Therapeutics Program, Fox Chase Cancer Center, Philadelphia, PA 19111, USA. ${ }^{4}$ Department of Oncology, Johns Hopkins University School of Medicine, Baltimore, MD 21287, USA. ${ }^{5}$ Department of Internal Medicine and Developmental Therapeutics Program, Yale Cancer Center, Yale School of Medicine, Yale University, New Haven, CT 06520, USA. ${ }^{6}$ Department of Otorhinolaryngology-Head and Neck Surgery, Inselspital, University Hospital and University of Bern, Bern 3010, Switzerland. Evgeny Izumchenko, Adrian D. Schubert and Michael J. Slifker contributed equally to this work. Correspondence and requests for materials should be addressed to

S.P. (email: suraj.peri@fccc.edu) or to E.A.G. (email: Erica.golemis@fccc.edu)
} 
S quamous cell carcinomas of the head and neck (SCCHN) are highly heterogeneous, arise from multiple anatomical sites, and are characterized by aggressive local invasion and overall poor prognosis. While oropharyngeal SCCHN is subclassified into human papillomavirus-initiated (HPV+) and HPVnegative (HPV-) disease, most HPV- cases arise from the oral cavity and larynx, with a smaller number of cases originating from the hypopharynx and oropharynx. Tobacco use and alcohol consumption are major risk factors for laryngeal carcinomas, which account for nearly $25 \%$ of SCCHN and are associated with high recurrence after treatment and limited overall survival (OS). Patients who are candidates for organ preservation are typically treated with a combination of chemotherapy and radiation, whereas those with high $\mathrm{T}$ stages often undergo surgery followed by adjuvant treatment.

Because treatments can be associated with significant morbidity, there is intense interest in identifying prognostic biomarkers and defining tumor subtypes that more accurately identify high-risk patients who may benefit from intensified therapy or novel targeted therapy trials. Molecular characterization of SCCHN to identify tumor subclasses associated with clinical outcomes ${ }^{1,2}$, using mutation ${ }^{3,4}$, epigenomic ${ }^{5,}$, , and expression profilingl, ${ }^{1}, 7$, including The Cancer Genome Atlas (TCGA $)^{8}$, has focused on tumors of the oral cavity and oropharynx, and the biology of HPV+ vs. HPV- disease ${ }^{1,2,9,10}$. The TCGA analysis identified laryngeal cancer subclasses, of atypical, classic, basal, and mesenchymal types based on mRNA profiling. However, these subclasses were not prognostic. Other studies using TCGA data have shown prognostic differences in SCCHN subtypes, including larynx, based on differences in intratumor heterogeneity ${ }^{10}$. However, no comprehensive analyses have identified prognostic molecular differences informative for the mechanism.
In this study, we analyzed data for SCCHN using a recently developed integrative clustering approach ${ }^{11}$ that simultaneously assesses DNA mutation, copy number variation (CNV), methylation, and gene expression, for 256 cases of SCCHN for which complete data for these parameters and clinical prognosis are available in the TCGA. This analysis identified two distinct clusters of laryngeal cancer, associated with strong prognostic value, and showed that mutations in the genes NSD1 and NSD2 entirely segregated to the cluster with favorable prognosis. Subsequent validation in an independent set of 63 laryngeal cancer samples again demonstrated that mutations in NSD1 or NSD2 are independent favorable prognostic biomarkers for laryngeal cancer.

\section{Results}

Integrative clustering of SCCHN. Using the methods recently described by Mo et al. ${ }^{11}$, we performed integrative analysis for 256 cases of SCCHN for which complete data on DNA mutation, $\mathrm{CNV}$, methylation, and gene expression were available in the TCGA. This distinguished 5 clusters that segregate tumors by disease site and HPV status (Fig. 1a; Supplementary Data 1, 2, and 3), and are associated with differences in OS (Fig. 1b). Overall, 73\% (25/34) of HPV+ tumors, predominantly derived from the oropharynx, fell in cluster 5 , marked by better prognosis (2-year OS $87 \%$, 95\% CI 68.8-95.0\%), as has been previously reported for this disease type ${ }^{8}, 9$. The 152 HPV- oral cavity tumors were found predominantly in clusters 1 (39\%) and 2 (37\%), with the remaining $24 \%$ distributed throughout clusters 3 , 4 , and 5. While TP53 mutations were predominant in oral and laryngeal clusters, mutation of TP53 was low in cluster $5(27 \%)$, reflecting the high incidence of HPV + oropharyngeal tumors, where TP53 is inactivated posttranslationally by the virally
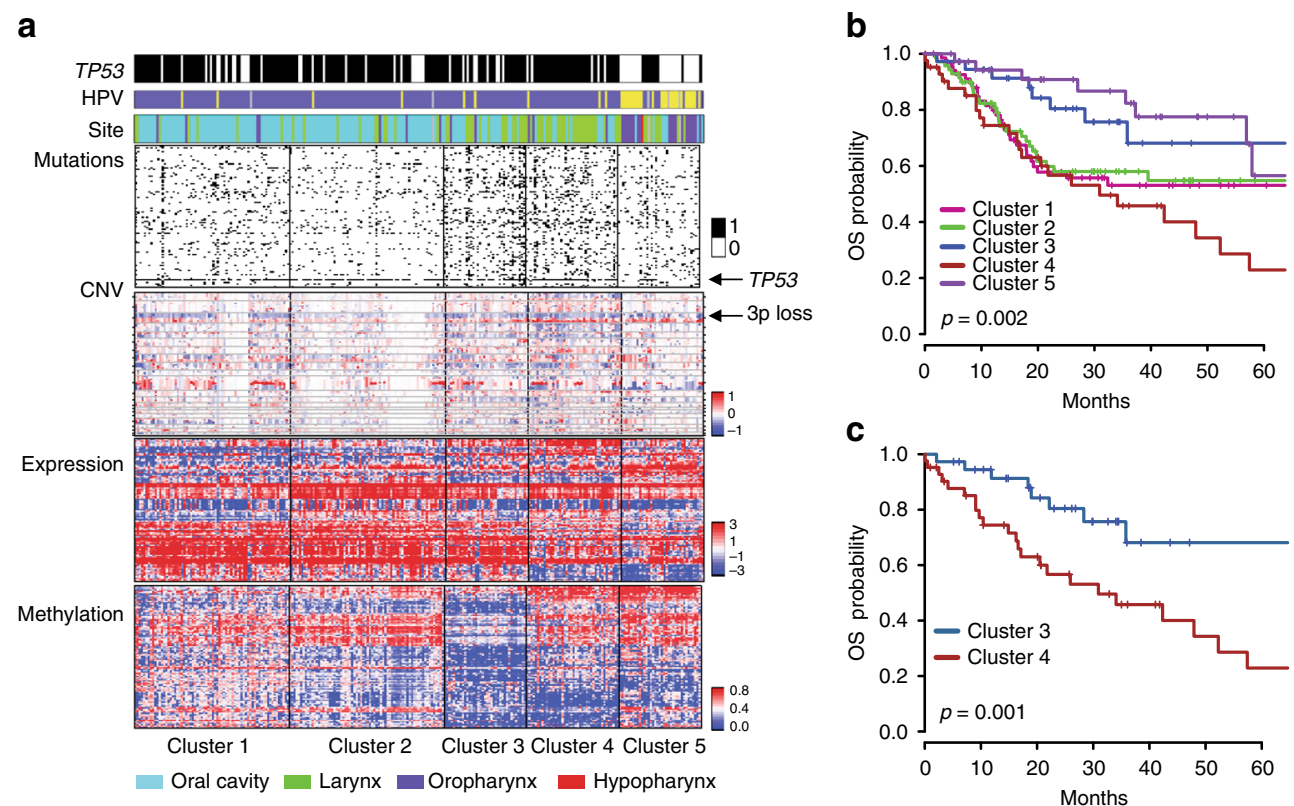

Fig. 1 Integrative clustering of 256 SCCHN tumors. a The top three rows indicate TP53 mutation (black), HPV status (purple = HPV-; yellow = HPV +; and gray $=$ unknown), and tumor site. Mutation panels indicate individual mutations in the top $25 \%$ most commonly mutated genes; note the marked mutational burden in clusters 3 and 4. CNV indicates the heatmap of chromosomal gains (red) and losses (blue). For gene expression, the heatmap indicates overexpression (red) or underexpression (blue) of genes; specific gene IDs are provided in Supplementary Data 1, and gene ontology (GO)enriched categories are provided in Supplementary Data 2. For methylation, a heatmap plots $\beta$ values of the probes as unmethylated (blue) or methylated (red). Genes corresponding to probes and enriched GO categories for methylation are listed in Supplementary Data 1 and 3, respectively. b Kaplan-Meier (KM) overall survival (OS) curves for all clusters identified in $\mathbf{a}$. The $p$-value (log-rank test) indicates a strong evidence against the null hypothesis that the risk of death is similar across all SCCHN clusters. c. KM OS curves for clusters 3 and 4 
a

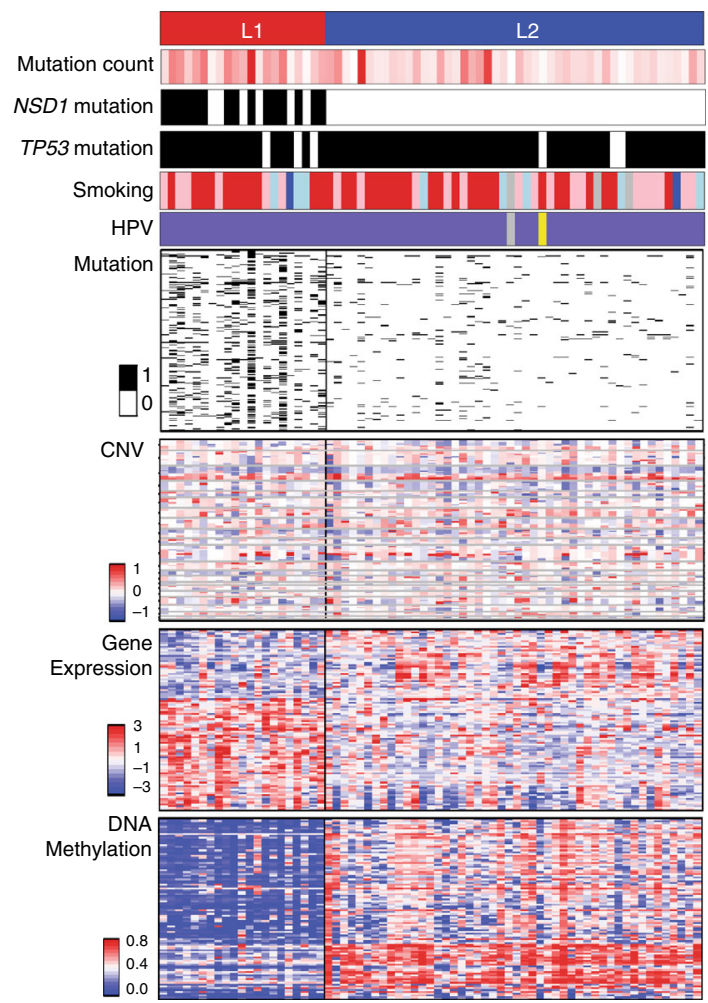

d

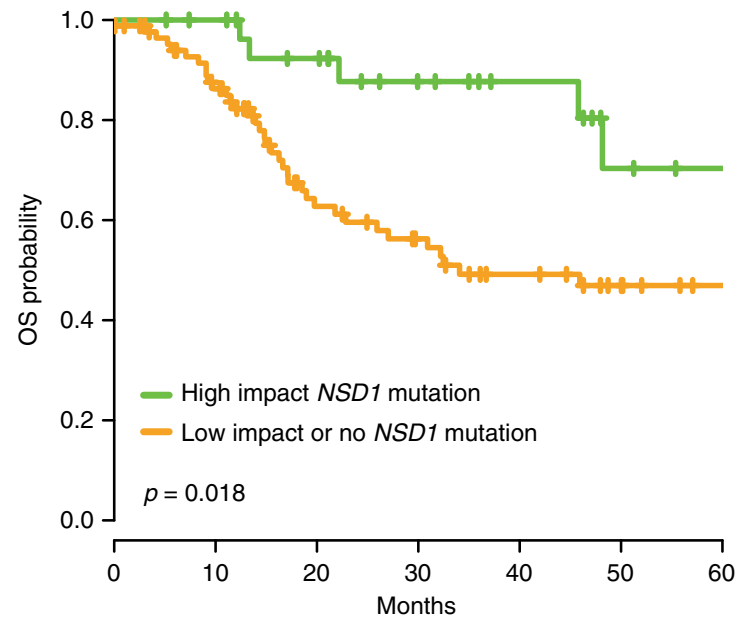

b

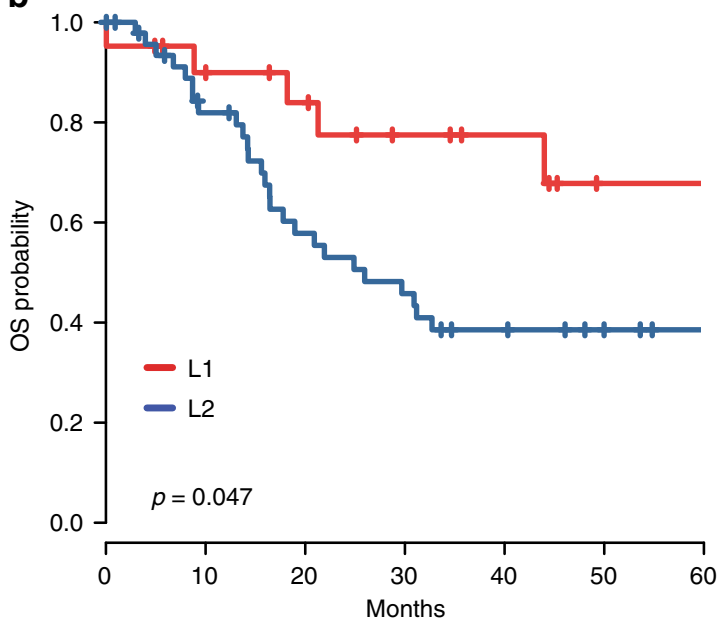

C

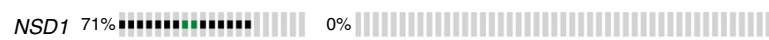

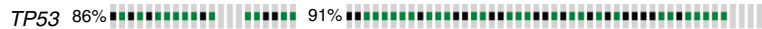

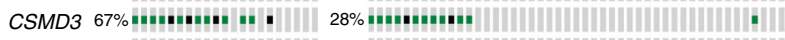

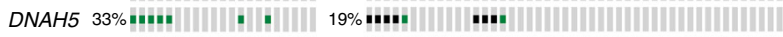

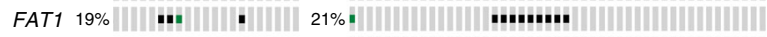

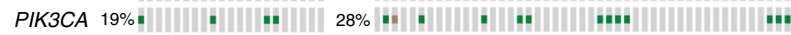

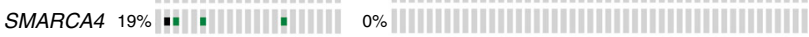

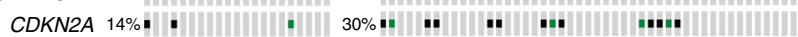

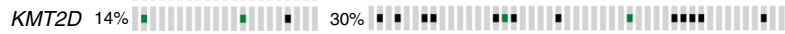

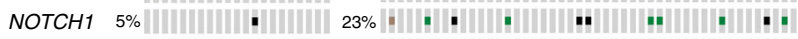

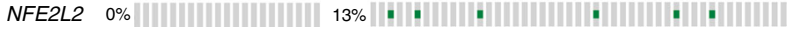

e

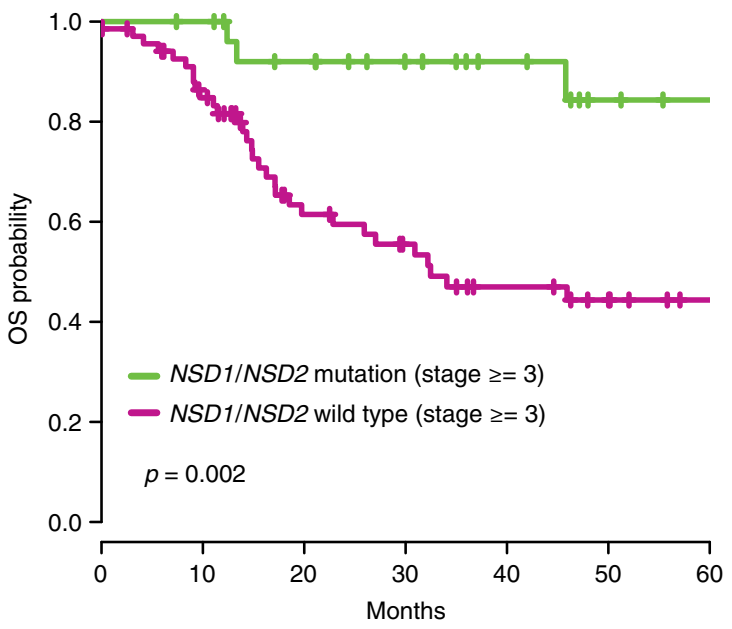

Fig. 2 Integrative clustering of 69 laryngeal tumors. a The top panel shows larynx L1 (red) and L2 (blue) clusters. Mutation count for each sample is provided as a color gradient. NSD1 and TP53 bars indicate mutations in these genes (black). Smoking status (red $=$ current smoker; pink $=$ former smoker < 15 years; cyan = former smoker $>15$ years; blue = lifelong nonsmoker; gray = status unavailable) and HPV status (purple = HPV-; yellow = HPV +; gray $=$ unknown) are indicated. Also see Fig. 1 Legend and Methods. b Kaplan-Meier curves depicting OS of L1 and L2 clusters ( $p$-value from log-rank test). c Mutation grid map of genes commonly mutated in L1 and L2. Each vertical bar represents a patient; black dot = truncating mutation; green = missense mutation. d KM curves for 116 laryngeal samples with NSD1 mutation impact determined by MutationAssessor ${ }^{39}$, Polyphen, and other function prediction algorithms. e KM curves for higher-stage laryngeal tumors (clinical stage 3 and 4) based on the presence or absence of predicted damaging mutations in NSD1 or NSD2

encoded E6 oncoprotein ${ }^{2}$. The recently described prognostic 3 pdel/TP53 subclass ${ }^{12}$ (Supplementary Fig. 1) is common in all HPV- clusters except cluster 2 (6\%).

Most laryngeal tumors segregated to clusters 3 (25\%) and 4 (39\%), with the remaining $36 \%$ distributed evenly across the other clusters. SCCHN tumors in cluster 3 were associated with significantly higher OS vs. those in cluster 4 (2-year OS: $81.6 \%$ (95\% CI 63.3-91.3\%) vs. 54.6\% (95\% CI 37.9-68.5\%); log-rank test: $p=0.001$; Fig. $1 \mathrm{c})$. This marked survival difference between laryngeal tumors in clusters 3 and 4 was intriguing, because the existence of two different laryngeal subtypes of prognostic value based on molecular profiles has not been previously described.

Identification of prognostic laryngeal cancer clusters. Given that laryngeal tumors were distributed into two major clusters with differences in OS, we repeated integrative clustering analysis focusing solely on the 69 laryngeal tumors, resulting in two 
distinct clusters, L1 and L2 (Fig. 2a). These clusters had a significant difference in survival, with a 2 -year recurrence-free survival (RFS) probability of $77.5 \%$ (95\% CI $50.1-91 \%)$ vs. $44.7 \%$ (95\% CI 29.6-58.7\%; log-rank test: $p=0.010$ ), and OS probability of $77.5 \%$ (95\% CI 50.1-91\%) vs. 53\% (95\% CI 37-66.7\%; log-rank test: $p=0.047$ ) (Fig. $2 \mathrm{~b}$ and Supplementary Fig. 2a). No significant differences in age, gender, race, TNM stage, or reported smoking or alcohol status distinguished the clusters (Supplementary Data 4, 5, 6, and 7). Cluster L2, associated with poor outcome, differed from cluster L1 in methylation (Supplementary Data 8 and 9) and gene expression (Supplementary Data 8 and 10 ), as well as lower mutation burden (mean 169.4 vs. 258.9 per tumor; Wilcoxon rank sum test: $p=0.014$ ), but not copy number changes. Among the genes reported as most commonly mutated in laryngeal cancer (including TP53, CDKN2A, PIK3CA, DNAH5, NOTCH, FAT1, NFE2L2, and NSD1), most of them were mutated at similar frequencies in L1 and L2 (Fig. 2c). However, NSD1 was a striking exception, with inactivating mutations limited to L1 (Fisher's exact test: $p<0.001$ ) (Fig. 2a, c and Supplementary Fig. 3a), and 15 of 21 tumors displaying such mutations. Further analysis of inactivating NSD1 mutations across the 5 SCCHN clusters indicated that these mutations concentrate in cluster 3 (Supplementary Data 4 and 5). Although NSD1 mutations were detected in oral tumors (Supplementary Data 11), the association of NSD1 mutation with better OS and RFS was specific to laryngeal tumors, suggesting a biological difference between tumor subsites (Supplementary Figs. $2 \mathrm{~b}$ and $3 \mathrm{~b}$ ).

Damaging mutations in NSD1 and NSD2 are prognostic of OS. We therefore analyzed the mutational profile of NSD1 in the complete TCGA set of 116 laryngeal tumors for which at least mutational data were available among a total of 526 SCCHN cases (Supplementary Data 4 and 5). Among NSD1 mutations in these tumors, 30 were predicted to be highly damaging based on multiple algorithms (Supplementary Data 5). In this expanded cohort, a significant association with improved OS (log-rank test: $p=0.018$ ) was seen for high-impact NSD1 mutations (2-year OS: $87.7 \%$; $95 \%$ CI $66.3-95.9 \%)$ in comparison to low-impact or no NSD1 mutations (2-year OS 59.6\%; 95\% CI 47.1-70\%) (Fig. 2d). We further extended this analysis, restricting investigation of OS to stage 3 and 4 tumors to increase specimen homogeneity, and also including consideration of the less-frequently mutated NSD1 paralog NSD2. This resulted in a striking increase in association between NSD1/2 mutation and OS, with a median OS of 95 months for patients with either gene mutated (2-year OS: $92 \%$; 95\% CI 71.6-97.4\%), vs. 32.5 months for those with both genes of wild type (2-year OS: 59.4\%; 95\% CI 45.6-70.8\%; log-rank test: $p$ $=0.002$ ) (Fig. 2e). In contrast, similar analysis of highly damaging NSD1 and NSD2 mutations in HPV- oral, oropharynx, and hypopharynx tumors did not reveal a significant association with OS (Fig. 3a). No significant association was observed between NSD1 or NSD2 mutational status and tumor stage in larynx (Fisher's exact test: $p=0.35$; Table 1 ) and oral tumors (Fisher's exact test: $p=0.3$ ). The results were specific to mutation rather than altered gene expression, as among cases with NSD1 and NSD2 wild-type alleles in oropharynx and hypopharynx tumors (Fig. 3b) and laryngeal tumors (Fig. 3c), NSD1 mRNA expression levels did not predict survival. Altogether, these observations that indicated loss-of-function mutations in NSD1 or NSD2 are associated with better prognosis specifically in laryngeal cancer.

To validate these results, we compiled a set of 63 previously unstudied laryngeal tumors from surgical resections obtained before any treatment, including chemotherapy or irradiation (Table 1). Using Fluidigm amplification and Illumina sequencing, we identified damaging mutations for NSD1 (21/63) (Fig. 4a) or a

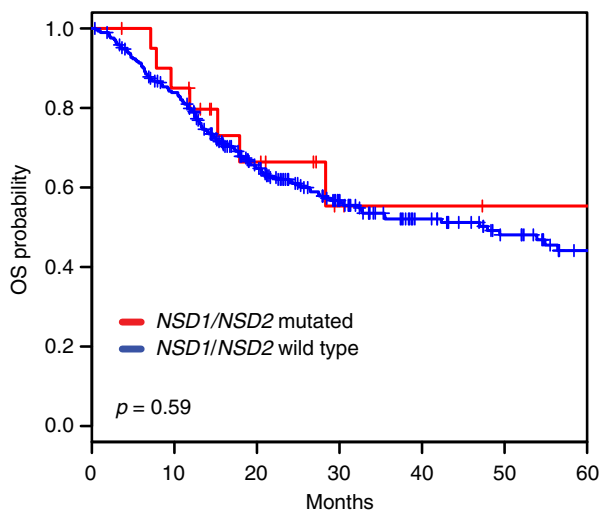

b

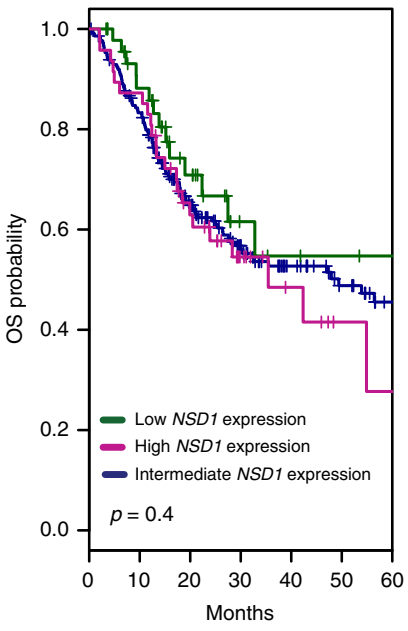

C

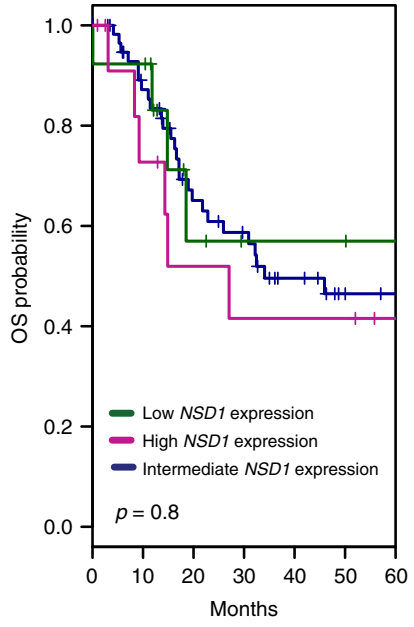

Fig. 3 Kaplan-Meier curves for overall survival (OS) based on NSD1/NSD2 mutation and NSD1 expression status in nonlaryngeal and laryngeal tumors, based on TCGA data. a Kaplan-Meier curves showing OS for nonlaryngeal HPV- (including oral, oropharynx, and hypopharynx) SCCHN. Red and blue curves show NSD1- and NSD2- mutated, and NSD1/NSD2 wild-type cases, respectively; OS differences are not significant ( $p$-value from log-rank test). b Kaplan-Meier curves showing OS differences for patients with NSD1 wildtype oral cancers trichotomized based on low ( $<$ or $15 \%$ quantile), intermediate ( $>15 \%$ and $<85 \%$ quantiles), or high ( $>85 \%$ quantile) NSD1 expression. c Kaplan-Meier curves showing OS for patients with NSD1/ NSD2 wild-type laryngeal cancers, with NSD1 expression trichotomized as in b

NSD2 (3/63) (Fig. 4b) in 24 of the 63 cases, with nonsense $(38 \%)$ and missense $(29 \%)$ mutations predominating. In addition to these, we identified nine patients with gene variants predicted to be nondamaging, with several known to be nonrare variants based on data in the Exome Aggregation Consortium $(\mathrm{ExAC})^{13}$ : we considered these as benign (Supplementary Data 12). The 24 patients with damaging NSD1 or NSD2 mutations had extended OS (2-year OS: $87.5 \%$; 95\% CI: 66.1-95.8\%) vs. the 39 with benign or no mutations (2-year OS: 61.5\%; 95\% CI: 44.5-74.7\%) (logrank test: $p<0.001$ ) (Fig. $4 c$ ). In extended comparison of the 116 TCGA specimens and of the 63 validation specimens with defined NSD1/NSD2 mutation status (Table 1), no significant differences in race, nodal status, or clinical stage distinguished patients with laryngeal tumors with or without these mutations. No significant association was found with age and gender in the TCGA cohort; interestingly, in the validation cohort, which contained a number of patients diagnosed at an earlier age, NSD1/2 mutation was associated with earlier diagnosis (Wilcoxon rank sum test: $p<$ 0.001 ) and gender (Fisher's exact test: $p=0.022$ ). 
Table 1 Results from statistical analyses for association between clinical characteristics and NSD1 and NSD2 (NSD) mutation status for laryngeal samples in the discovery (TCGA) and validation cohorts

\begin{tabular}{|c|c|c|c|c|c|c|c|c|c|c|c|}
\hline & & \multicolumn{5}{|c|}{ TCGA } & \multicolumn{5}{|c|}{ Validation } \\
\hline & & \multicolumn{2}{|c|}{$\begin{array}{l}\text { NSD1/2 mutated, } \\
n=32\end{array}$} & \multicolumn{2}{|c|}{$\begin{array}{l}\text { Wild type, } \\
n=84\end{array}$} & \multirow[t]{2}{*}{$p$-value ${ }^{\star}$} & \multicolumn{2}{|c|}{ NSD1/2 mutated, $n=24$} & \multicolumn{2}{|c|}{ Wild type, $n=39$} & \multirow[t]{2}{*}{$p$-value } \\
\hline & & $n$ & $\%$ & $n$ & $\%$ & & $n$ & $\%$ & $\mathbf{n}$ & $\%$ & \\
\hline \multicolumn{12}{|l|}{ Patient Characteristics } \\
\hline \multirow{5}{*}{$\begin{array}{l}\text { Age, yrs Median (range) } \\
\text { Age, yrs group }\end{array}$} & & \multicolumn{2}{|c|}{$61.5(38-80)$} & \multicolumn{2}{|c|}{$62(39-83)$} & 0.51 & \multicolumn{2}{|c|}{$51.5(36-86)$} & \multicolumn{2}{|c|}{$65(44-83)$} & $<0.001$ \\
\hline & & & & & & 0.49 & & & & & $<0.001$ \\
\hline & $36-49$ & 4 & 12.5 & 7 & 8.3 & & 11 & 45.8 & 2 & 5.1 & \\
\hline & $50-69$ & 24 & 75.0 & 59 & 70.2 & & 10 & 41.7 & 25 & 64.1 & \\
\hline & $70-86$ & 4 & 12.5 & 18 & 21.4 & & 3 & 12.5 & 12 & 30.8 & \\
\hline \multirow[t]{3}{*}{ Gender } & & & & & & 1.00 & & & & & 0.022 \\
\hline & Female & 5 & 15.6 & 15 & 17.9 & & 9 & 37.5 & 4 & 10.3 & \\
\hline & Male & 27 & 84.4 & 69 & 82.1 & & 15 & 62.5 & 35 & 89.7 & \\
\hline \multirow[t]{4}{*}{ Race } & & & & & & 0.15 & & & & & 0.88 \\
\hline & Black & 2 & 6.3 & 17 & 20.2 & & 5 & 20.8 & 7 & 17.9 & \\
\hline & White & 29 & 90.6 & 62 & 73.8 & & 18 & 75.0 & 31 & 79.5 & \\
\hline & Other/unknown & 1 & 3.1 & 5 & 6.0 & & 1 & 4.2 & 1 & 2.6 & \\
\hline \multirow[t]{5}{*}{ Smoking status } & & & & & & 0.34 & & & & & \\
\hline & Current & 19 & 59.4 & 39 & 46.4 & & smo & a unavailable & & & \\
\hline & Former & 13 & 40.6 & 36 & 42.9 & & & & & & \\
\hline & Never & 0 & 0.0 & 6 & 7.2 & & & & & & \\
\hline & Unknown & 0 & 0.0 & 3 & 3.6 & & & & & & \\
\hline \multirow[t]{4}{*}{ Alcohol use } & & & & & & 0.75 & alco & unavailable & & & \\
\hline & Yes & 23 & 71.9 & 53 & 63.1 & & & & & & \\
\hline & No & 9 & 28.3 & 29 & 34.5 & & & & & & \\
\hline & Unknown & 0 & 0.0 & 2 & 2.4 & & & & & & \\
\hline \multicolumn{12}{|l|}{ Tumor Characteristics } \\
\hline \multirow[t]{5}{*}{ Clinical stage } & & & & & & 0.35 & & & & & 0.72 \\
\hline & Stage I,II & 4 & 12.5 & 12 & 14.3 & & 5 & 20.8 & 12 & 30.8 & \\
\hline & Stage III & 11 & 34.4 & 16 & 19.1 & & 7 & 29.2 & 7 & 18.0 & \\
\hline & Stage IV & 16 & 5.0 & 53 & 63.1 & & 10 & 41.7 & 17 & 43.6 & \\
\hline & Stage unknown & 1 & 3.1 & 3 & 3.6 & & 2 & 8.3 & 3 & 7.7 & \\
\hline \multirow[t]{4}{*}{ Node status } & & & & & & 0.67 & & & & & 0.58 \\
\hline & Node Neg (NO) & 18 & 58.1 & 39 & 49.4 & & 12 & 54.5 & 23 & 63.9 & \\
\hline & Any positive $(\mathrm{N}+)$ & 13 & 41.9 & 40 & 50.6 & & 10 & 45.5 & 13 & 36.1 & \\
\hline & Unknown ${ }^{a}$ & 1 & & 5 & & & 2 & & 3 & & \\
\hline
\end{tabular}

One study has suggested a relationship between NSD1 depletion, DNA hypomethylation, and differential expression of some miRNAs ${ }^{8}$, including enriched expression of miR-200a/b, which is involved in epithelial-mesenchymal transition (EMT) ${ }^{14}$. However, that work analyzed a combined set of oral and laryngeal tumors. Considering only the laryngeal tumor set, we find several miRNAs to be differentially expressed between L1 and L2 (Fig. 5a). However, no selective enrichment for miRNAs associated with EMT distinguished these clusters. Further, enrichment analysis for an $\mathrm{EMT}^{15}$ mRNA signature showed comparable enrichment in L1 and L2 (Fig. 5b, c), likely reflecting the advanced stage of most laryngeal specimens in the TCGA. These results bear further investigation in an extended laryngeal cohort for which mRNA and miRNA data are available, since as an important caveat, the lack of significant association might, in part, represent the limited statistical power available when considering only laryngeal cases. However, extending our analysis, we carried out Gene Set Enrichment Analysis (GSEA) ${ }^{16}$ comparing genes differentially expressed between L1 and L2 (differing by 775 genes with DESeq2 Wald test ${ }^{17}: p<0.001$; Supplementary Data 13). Interestingly, this identified several gene sets (Supplementary Fig. 4 and Supplementary Data 14) involved in stem cell maintenance and hypoxic response that are elevated in L2, compatible with the poorer overall survival of patients in this class.

\section{Discussion}

Management of laryngeal cancer is complex where therapeutic resistance contributes to significant morbidity. Moreover, in patients that are candidates for total laryngectomy have decreased quality of life. Therefore, prognostic biomarkers for therapeutic intensity and predictive biomarkers for radiosensitivity (and thus suitability for larynx preservation) are urgently needed. Using integrative clustering of four different genomic data of SCCHN including mutation, copy number, gene expression, and methylation obtained from TCGA, we identified a novel laryngeal cluster with a significant difference in OS where the prognostic laryngeal cancer subtype is associated with mutations in NSD1 and NSD2.

Inherited mutations in NSD1 cause Sotos syndrome ${ }^{18}$, and are implicated in a number of cancers ${ }^{19}$, as is mutation of $N S D 2^{20}$. As lysine methyltransferases, NSD1 and NSD2 influence gene expression by methylating specific histone sites (H3K36, H4K20) and nonhistone targets such as $N F-\kappa B^{19,21,22}$. Mutations in NSD1 have been associated with extensive demethylation of DNA in CpG islands at promoters, as well as intergenic regions ${ }^{8}, 23$, compatible with the significantly lower levels of methylation observed in cluster L1 (Fig. 2a, Supplementary Data 15 and 16). Using germline DNA from Sotos syndrome patients, it has been shown that loss of NSD1 function is associated with extensive DNA hypomethylation ${ }^{24}$. H3K36 methylation is known to induce 
a

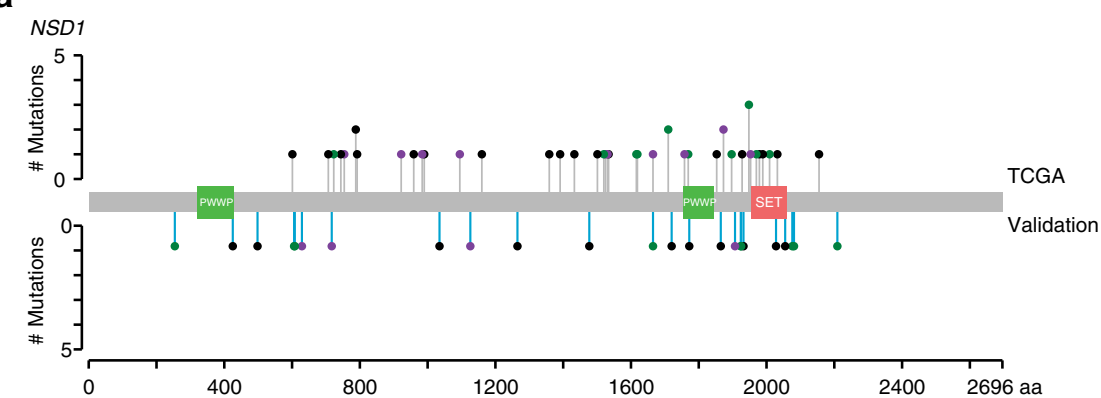

b

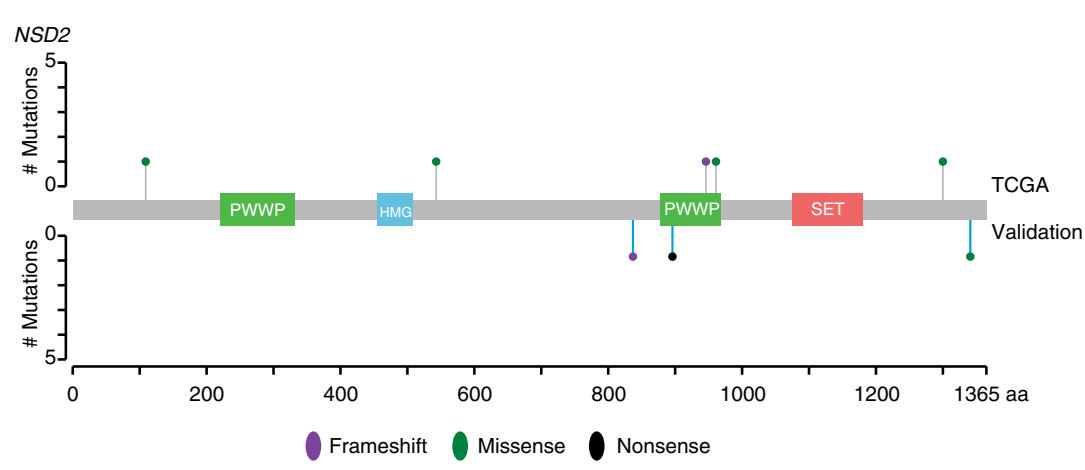

C

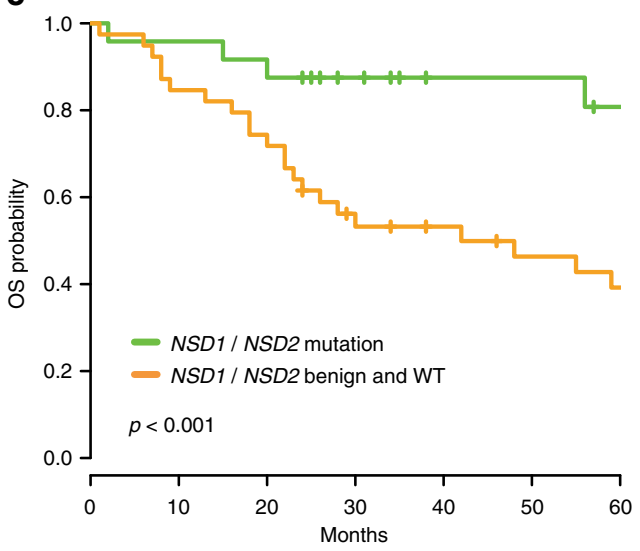

Fig. 4 Validation of NSD1 and NSD2 mutations in an independent laryngeal cancer cohort. a Plot indicates the location of nonsense (black), frameshift (purple), and missense (green) mutations affecting the NSD1 protein. NSD1 functional domains are indicated. Lollipops above the line indicate mutations reported in the TCGA. Lollipops below the line depict mutations identified in the validation cohort. b Plot similar to a, for NSD2. c Kaplan-Meier curve indicates OS of patients with laryngeal tumors with damaging NSD1 or NSD2 mutation $(n=24)$ or without such mutations $(n=39)$, in the validation cohort ( $p$-value from log-rank test)

localized activity of DNA methyltransferases ${ }^{25-27}$; for example, the de novo DNA methyltransferase DNMT3B is recruited to gene body regions that are enriched for $\mathrm{H} 3 \mathrm{~K} 36 \mathrm{me} 3$ by SETD2 $2^{25}$, 28. Interestingly, a recent publication that showed decreased dimethylation of lysine in $\mathrm{H} 3$ histones (H3K36me2) in cases of SCCHN was associated with damaging NSD1 mutations ${ }^{29}$, although this study did not identify a prognostic role for NSD1 mutations, consider a possible role for NSD2, or assign prognostic value specifically to tumors of the larynx. While they have been reported as tumor suppressive in neuroblastoma and glioma ${ }^{30}$, amplification and overexpression of NSD1 have been reported as oncogenic in some settings, suggesting context-dependent action $^{31}$. While other studies $1,3,4,8$ have cataloged distinct prognostic molecular features for $\mathrm{HPV}+$ and oral tumors, this work for the first time distinguishes molecular subclasses of laryngeal tumors with markedly different prognosis, with a positive outcome specifically associated with mutation of NSD1 and NSD2. This classification markedly differs from earlier TCGA analyses, which segregated laryngeal tumors into non-prognostic "classical" and "atypical" subclasses lacking the separation of $N S D 1^{8}$ (Fig. 6; Table 2). Developmentally, the larynx differs significantly from the oral cavity, with the larynx, trachea, and lung arising from the endoderm and a common lung bud, while the oral cavity and hypopharynx arise from a complex interplay of ectodermal and endodermal lineages ${ }^{32}$. Such lineage differences may underlie distinct activities of NSD1 and NSD2 in these SCCHN settings.

The fact that mutations associated with hypomethylation of histones and DNA is provocative for laryngeal cancer, given the emergence of DNA demethylating agents such as azacitidine (5aza-CR) and decitabine (5-aza-CdR) as promising agents in clinical trials ${ }^{33}$. Our data suggest that demethylating agents and EZH2 inhibitors may be particularly effective in tumors lacking NSD1 or NSD2 mutations, and clinical trials seeking to establish survival benefits for these drugs in patients with laryngeal tumors should restrict study populations to those with NSD wild-type tumors. 
a

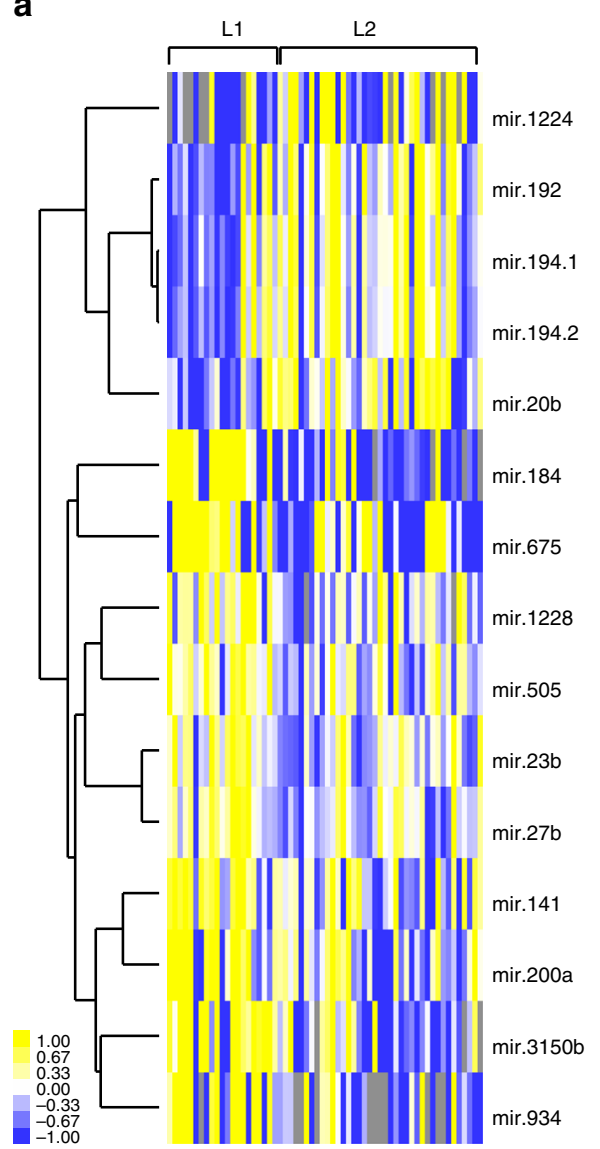

b
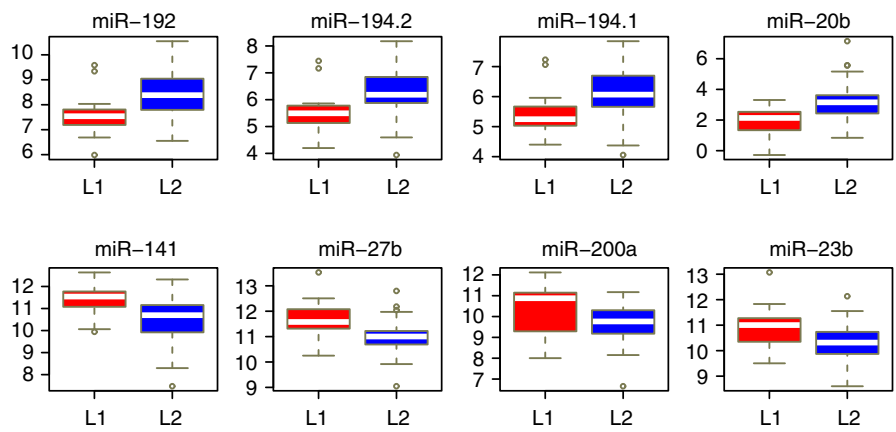

C

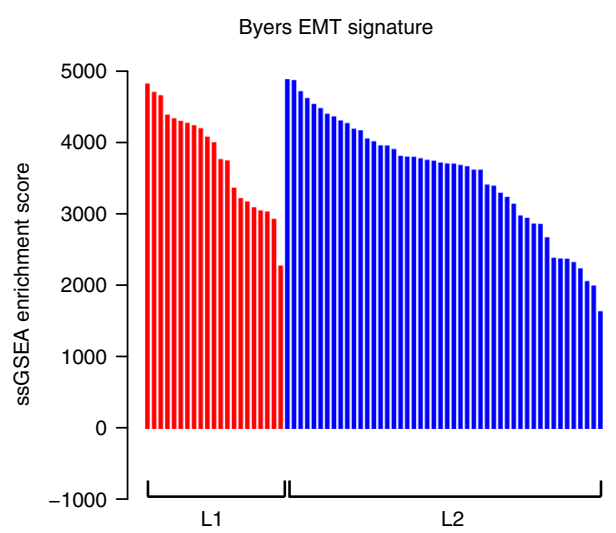

Fig. 5 Enrichment profiles of EMT signatures and microRNAs in L1 and L2. a Heatmap showing miRNAs with significant differences in expression profile between the L1 and L2 clusters. Blue indicates underexpression, yellow indicates overexpression, and gray indicates expression data not available. b Box plot showing the normalized gene counts (reads per million miRNA mapped) of miRNAs that are over- or underexpressed in L1 clusters of laryngeal tumors. $X$-axis indicates the L1 and L2 clusters, while the $Y$-axis indicates normalized gene counts of miRNA for both L1 (blue box) and L2 (red box) samples. c Waterfall plot depicting the enrichment score for each laryngeal tumor against a gene set of 76 genes forming an EMT signature as defined by Byers et al. ${ }^{15}$, using the ssGSEA algorithm. Blue and red bars indicate tumors in L1 and L2 clusters, respectively. Y-axis shows the enrichment scores. A positive enrichment score indicates the extent to which genes in EMT signature are overexpressed in each tumor sample. Similarly, a negative enrichment score indicates the extent to which genes in EMT signature are downregulated in the tumor sample

Also of potential clinical relevance, the methyltransferase EZH2 selectively methylates $\mathrm{H} 3 \mathrm{~K} 27$, and several clinical trials are evaluating the efficacy of EZH2 inhibition in treating solid tumors and hematologic malignancies ${ }^{34,35}$. Although the use of EZH2 inhibitors in the context of NSD mutations has not been studied, one recent report suggested a promising mechanism for application of EZH2 inhibitors in NSD2-overexpressing myeloma cells ${ }^{36}$.

Our study suggests that NSD mutation status may play an important role in the efficacy of such inhibitors in patients with laryngeal cancer. Finally, although no significant association between clinical parameters (i.e., tumor stage and nodal status) and NSD1/2 mutation status was observed in either the discovery or validation sets, further evaluation in additional independent cohorts is required. In sum, this work nominates NSD1 and NSD2 as rapidly exploitable prognostic biomarkers and targets for functional scrutiny in laryngeal cancers.

\section{Methods}

Clustering of molecular data for all SCCHN cases. Molecular data sets describing somatic mutations, CNV, gene expression based on RNA-seq, and methylation for 256 SCCHN cases were obtained from the TCGA data portal (https://tcga-data.nci.nih.gov/tcga/) (August 2014 data freeze) and The Cancer Genome Atlas Genome Data Analysis Center (GDAC) Firehose website (https:// confluence.broadinstitute.org/display/GDAC/). To maintain coherency of analysis across different data layers and cancer types, we used normalized data classified as levels 2 and 3 in the TCGA data hierarchy ${ }^{37}$ as the starting point of our analysis. Of the 528 samples that are available from TCGA consortium, 256 samples had profiling data for all 4 genomic data sets (mutations, CNV, gene expression, and methylation), and were used for integrative clustering.

For somatic mutation data, all genes with nonsynonymous or nonsense variants from among these 256 cases were identified and used to construct a binary matrix after applying a $5 \%$ mutation frequency cutoff to remove infrequently mutated genes. For copy number variation data, we applied the strategy described in the supplement of Mo et al. ${ }^{11}$ to obtain a set of nonredundant regions and representative copy number quantifications for each region. Germline copy number variants and regions from chromosomes $\mathrm{X}$ and $\mathrm{Y}$ were removed from the data using standard approaches ${ }^{38}$. For detailed analysis to identify NSD1 missense variants as deleterious or benign, we used the MutationAssessor/Functional Impact Score (FIS) ${ }^{39}$, Polyphen, and a Grantham score available through SeattleSeq annotation server (http://snp.gs.washington.edu/SeattleSeqAnnotation138/). MutationMapper ${ }^{40}$ was used to display mutations graphically. For analysis of OS associated with NSD1 mutation in larynx cancer, the full set of 116 laryngeal tumors present in the 526-specimen SCCHN TCGA collection containing mutational data was analyzed.

For gene expression data, gene-level RPKM values (reads per kilobase per million mapped reads) from the TCGA data portal were $\log _{2}$-transformed and subjected to a variation filter: the 5000 genes with the highest median absolute deviation (MAD) across all 256 SCCHN specimens were retained. Similarly, level 3 methylation data ( $\beta$ values from the Illumina HumanMethylation450k bead chip) were filtered to retain 2000 probes with the highest MAD across samples. These $\beta$ values were then logit-transformed into $\mathrm{M}$-values ${ }^{41}$.

The resulting four matrices were used as input to integrative clustering analysis using iClusterPlus ${ }^{11}$. A plot relating the percentage of explained variation to the number of clusters suggested five clusters as an appropriate choice for clustering 


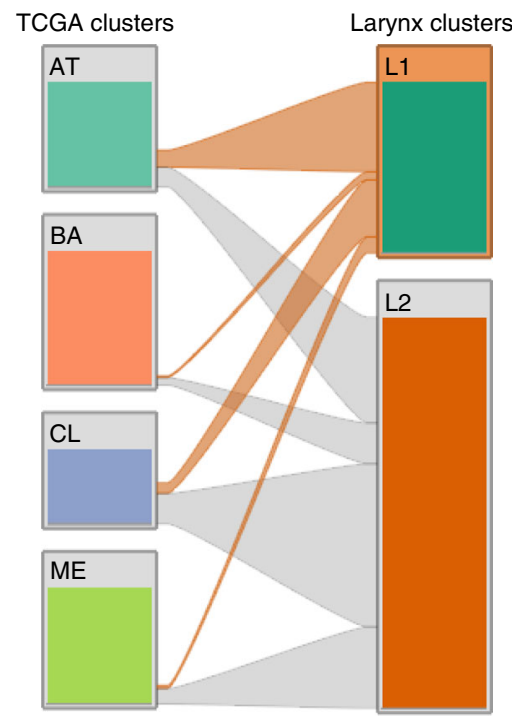

Fig. 6 Relationship between SCCHN TCGA (mRNA derived) subtypes, and integrative clustering-derived laryngeal subtypes assigned in this study. The left column represents the distribution of laryngeal tumors among the 256 SCCHN specimens (laryngeal, oral, oropharynx, and hypopharynx) assigned by the TCGA as atypical (AT), basal (BA), classical (CL), and mesenchymal (ME) subtypes. Gray and orange bands indicate the redistribution of TCGA laryngeal tumors to the L1 and L2 prognostic clusters defined in this work (right). The plot shows that the majority of tumor samples in L1 cluster belong to the atypical (52\%) and classical (33\%) subtypes, whereas the majority of $\mathrm{L} 2$ tumor samples belong to classical (42\%), atypical (27\%), and mesenchymal (21\%) subtypes

the 256 SCCHN samples (Supplementary Fig. 5a). To analyze segregation of 3p loss and TP53 mutation among the clusters, a median of the segmentation mean (MSM) values between chromosome 3p25 and 3p13 was calculated. Cases with MSMs $<-0.3^{42}$ are identified as cases with $3 p$ loss. These samples were further segregated into two classes based on the mutant and wild-type status of TP53 gene.

Clustering of data to define $\mathbf{L 1}$ and $\mathbf{L 2}$ classes. We also applied the methods described above to cluster the 69 laryngeal samples among the 256 fully characterized SCCHN samples. The processing of the data described above for expression, CNV, and methylation was applied to larynx samples. A submatrix of the SCCHN mutation matrix representing the larynx samples was extracted. The resulting data matrices were analyzed using iClusterPlus; given the relatively small sample size, a two-cluster solution was selected (Supplementary Fig. 5b). The resulting data were depicted as a heatmap showing mutation, copy number, gene expression, and methylation. The mutation counts were shown as a gradient where red and white indicate maximal (1463) and minimal mutation counts (3) (Fig. 2a). The mutational burden between L1 and L2 clusters was compared by applying Wilcoxon rank sum test.

In order to identify methylation differences distinguishing the L1 and L2 laryngeal clusters, we applied the following data-processing steps. First, probes with all $\beta$ values less than 0.1 were removed. Next, probes were mapped to $158,848 \mathrm{CpG}$ islands and 6127 promoter sites, and were aggregated to obtain median M-values for each such region. These steps were performed in R using the Bioconductor package wateRmelon ${ }^{43}$. Subsequently, we applied Wilcoxon rank sum tests to compare methylation levels between L1 and L2. FDR-adjusted $p$-values were calculated with the Benjamini-Hochberg method ${ }^{44}$, and a 5\% FDR cutoff was applied to select differentially methylated regions.

We identified differentially expressed genes between clusters L1 and L2 using DESeq $2^{17}$, which models RNA-seq read counts with a negative binomial distribution. A total of 775 genes were identified as differentially expressed (Wald test $p<0.001$ and $\log 2$-fold change $>1$ and $<-1$ ) and were used for enrichment analysis with Gene Set Enrichment Analysis (GSEA) ${ }^{16}$, which was run using default parameters and 'classic' as the enrichment statistic. For NSD1 wild-type SCCHN tumors, to evaluate whether NSD1 gene expression was associated with survival differences in laryngeal or oral cancer cases, we used Voom-normalized ${ }^{45}$ RSEM (RNA-Seq by Expectation Maximization) expression data. NSD1 expression was trichotomized into low ( $<$ or $=15 \%$ quantile), intermediate $(>15 \%$ and $<85 \%$ quantile), or high ( $>$ or $=85 \%$ quantile), and survival distributions were estimated and compared using Kaplan-Meier method and log-rank test, respectively, on the resulting groups.

\section{Table 2 Relationship between SCCHN TCGA (mRNA derived) subtypes, and integrative clustering-derived laryngeal subtypes assigned in this study}

\begin{tabular}{lll} 
TCGA clusters & L1 larynx cluster & L2 larynx cluster \\
\hline Atypical (AT) & $11(52 \%)$ & $13(27 \%)$ \\
Basal (BA) & $1(5 \%)$ & $5(10 \%)$ \\
Classical (CL) & $7(33 \%)$ & $20(42 \%)$ \\
Mesenchymal (ME) & $2(10 \%)$ & $10(21 \%)$
\end{tabular}

Numbers of samples and percentage of intersection between laryngeal clusters and TCGA clusters

We evaluated the differential miRNA expression between L1 and L2 clusters based on miRNA quantification reported as reads per million miRNA mapped, establishing significance by applying Wilcoxon rank sum tests. FDR-adjusted $p$ values were calculated with Benjamini-Hochberg method, and a 5\% FDR cutoff was applied to select differentially expressed miRNAs. To visualize the differentially expressed miRNAs, we applied hierarchical clustering on miRNA expression data using average linkage clustering with Pearson's uncentered correlation as a distance measure. The resulting clustered data table was used as input to Java TreeView (http://jtreeview.sourceforge.net) to generate a heatmap. In order to assess the enrichment of epithelial-mesenchymal transition signatures in L1 and L2 clusters, we applied single-sample GSEA (ssGSEA) ${ }^{46}$ using the EMT signatures curated from Byers et al. ${ }^{15}$ and the available gene sets from MSigdb resource. The resulting enrichment scores were depicted as waterfall plots.

The functional significance of genes that contributed to segregation of five SCCHN and two laryngeal clusters was assessed using enrichment of gene ontology terms (GO) (biological process and molecular function) present in the DAVID bioinformatics resources $^{47}$ (Supplementary Data 2,3,8, and 9). To identify and depict the relationship between tumors from L1 and L2 clusters and gene expression- derived subtypes defined by TCGA, we obtained 279 tumor class labels indicating the subtypes from TCGA ${ }^{8}$. We stratified the relationship between these two groups using StratomeX tool ${ }^{48}$. In a StratomeX plot (Fig. 6), columns indicate TCGA mRNA classification (atypical, basal, classical, and mesenchymal) and laryngeal L1 and L2 tumors. The bands between these two groups indicate the intersection of tumor samples between two groups. The width of the band shows the level of intersection.

Validation of NSD1/2 mutations and prognostic significance. A validation set for laryngeal tumors was compiled from the biosample registries of Sidney Kimmel Cancer Center at Johns Hopkins School of Medicine (JH) and Fox Chase Cancer Center (FCCC) (with clinical characteristics summarized in Table 1). Study protocols from both institutions were reviewed and approved by the institutional review board (IRBs) from both institutions, and informed consent was received from all human patients included in the study. All tumors from $\mathrm{JH}$ cohort were fresh frozen tumors, while FCCC samples were formalin-fixed paraffin embedded (FFPE). All patients who were treated with chemotherapy or radiotherapy prior to their surgery were excluded, as were tumor samples from patients with synchronous diseases including esophageal and lung cancers. We included those cases for which definitive dates for diagnosis, treatment modalities, disease recurrence or metastasis, and time of death or last contact were available. A total of 99 samples were identified from both $\mathrm{JH}(n=68)$ and FCCC $(n=31)$ biosample repositories for evaluation of NSD1/2 mutation status. Based on exclusion criteria, we excluded 15 and 7 samples from JH and FCCC cohort from the analysis, respectively. Samples with low DNA yield or quality were eliminated (JH: $n=3$; FCCC: $n=11$ ). After accounting for exclusion and inclusion criteria listed above, we obtained 63 (JH: $n=50$; FCCC: $n=13$ ) samples from both institutions. All samples were reviewed by a pathologist to reconfirm the diagnosis prior to DNA extraction. Frozen tissues were cut into $5-\mu \mathrm{m}$ sections, stained with H\&E, and examined by light microscopy. Lesions with a low neoplastic cellularity $(<70 \%)$ were additionally microdissected to remove contaminating normal cells before DNA extraction. Paraffin-embedded slides were microdissected to obtain $>70 \%$ neoplastic cells. Neoplastic cellularity was estimated from the sequential slides, which highly reflect the cellularity of the section used for DNA sequencing.

DNA isolation. Genomic DNA was isolated from fresh frozen samples by the QIAamp DNA Kit (Qiagen). DNA extractions from FFPE-derived material were performed using standard protocols as previously described ${ }^{49}$. All samples were quantified using a DeNovix NanoDrop spectrophotometer (Wilmington, DE).

NSD1/NSD2 amplification. Primers pairs (91 for NSD1 and 49 for NSD2) were designed by Fluidigm (San Francisco, USA) to cover all exons of the NSD1/NSD2 genes and exon-intron boundaries. A total of $100 \mathrm{ng}$ of genomic DNA from neoplastic lesions were used for PCR amplification of target genes using an Access Array ${ }^{\text {sa }}$ microfluidic chip according to the manufacturer's instructions. Each sample 
was combined with primer pairs in a microfluidic chip, and thermal cycling on a Fluidigm FC1 Cycler was performed. PCR products were then collected using the integrated fluidic circuit controller and transferred to a 96-well plate. In a separate PCR, Illumina sequence-specific adaptors and barcodes were attached. Prior to sequencing, all amplified samples were checked on the Agilent 2100 BioAnalyzer to determine if the PCR product in the DNA reaction has the expected size. All fresh frozen samples passed the quality control (QC). Twelve FFPE-derived samples did not pass the QC and were excluded from sequencing. A total of 50 fresh frozen and 13 FFPE cases were considered for subsequent downstream analysis.

Sequencing. Pooled and indexed PCR products were sequenced on the Illumina MiSeq instrument following standard protocols with the following modifications: Illumina-specific sequencing primers were substituted with a mixture of two Fluidigm-specific primer pairs (FL1 and FL2). Quality and coverage metrics for MiSeq sequencing data indicated a mean coverage depth of 2000 reads. The sequencing reads were aligned to human genome (Hg19) using BWA ${ }^{50}$. The resulting BAM files were processed using the GATK best-practices pipeline $e^{51}$ with no mark duplicate step. We applied the Mutect2 algorithm (based on Mutect ${ }^{52}$ ) with default parameters using the tumor samples-only mode. Variants were filtered at an $8 \%$ variant allele fraction ${ }^{53}$. The resulting VCF files were processed using ANNOVAR $^{54}$ for functional annotation of identified variants, and with independent variant calling using HaplotypeCaller (GATK) and VarScan ${ }^{55}$ for comparison. Most of the NSD1/2 damaging variants were identified by all three variant callers. In assessing the functional impact of missense variants, we applied a rule that at least 3 functional impact prediction algorithms (viz. SIFT ${ }^{56}$, Polyphen $2^{57}$, LRT ${ }^{58}$, Mutation Assessor ${ }^{39}$, and FATHMM ${ }^{59}$ ) predict functionally damaging variants. For predicting overall survival differences among NSD1/2 loss-of-function mutations, we combined benign- and wild-type alleles into one group.

Survival analyses. Clinical information including follow-up and new tumor event (NTE) data for 526 SCCHN samples (including the 256 samples chosen for clustering) were obtained from the TCGA website (July 2015). OS and RFS distributions were estimated using Kaplan-Meier methods. OS was based on vital status and "days to death" (CDE_ID:3165475) from initial pathologic diagnosis. Individuals who were still alive at the time of the last follow-up were censored. The RFS outcome included time to first recurrence or death (with an individual censored if neither event was observed prior to the last follow-up). Recurrence was defined as the appearance of either "locoregional recurrence" or "distant metastasis", and time to first recurrence was estimated from "days to new tumor event after initial treatment” (CDE_ID: 3392464). As this item starts at the end of initial treatment rather than the date of pathologic diagnosis, the recurrence-free interval may be underestimated; data on the interval from diagnosis to initial treatment were not available in this data freeze. Survival curves were compared with log-rank tests, and these calculations were done using the $\mathrm{R}$ 'survival' package ${ }^{60}$. To test the association between clinical parameters (and the presence of mutations) and cluster membership, Fisher's exact or Wilcoxon rank sum tests were used. These tests were two-sided with 5\% type 1 error. Wherever necessary, significance levels of multiple comparisons were corrected for type 1 error rates using Benjamini-Hochberg falsediscovery rate (FDR) method ${ }^{44}$. To test the association between clinical parameters (and the presence of mutations) and cluster membership, or the presence/absence of damaging mutations of NSD1 or NSD2 genes, two-sided Fisher's exact and Wilcoxon rank sum tests were used, and $p$-values $<0.05$ were considered significant.

Data availability. De-identified patient variant data that support the findings of this study are available from the corresponding authors upon request, and are submitted to the database of Genotypes and Phenotypes (dbGaP). Primary results from data analyses were done using standard $\mathrm{R}$ and Bioconductor packages including iClusterPlus and Survival. R scripts calling routines from these packages will be available from the corresponding author upon request.

Received: 12 November 2015 Accepted: 19 October 2017

Published online: 24 November 2017

\section{References}

1. Keck, M. K. et al. Integrative analysis of head and neck cancer identifies two biologically distinct HPV and three non-HPV subtypes. Clin. Cancer Res.: Off. J. Am. Assoc. Cancer Res. 21, 870-881 (2015).

2. Klussmann, J. P. et al. Genetic signatures of HPV-related and unrelated oropharyngeal carcinoma and their prognostic implications. Clin. Cancer Res.: Off. J.Am. Assoc. Cancer Res. 15, 1779-1786 (2009).

3. Agrawal, N. et al. Exome sequencing of head and neck squamous cell carcinoma reveals inactivating mutations in NOTCH1. Science 333, 1154-1157 (2011).

4. Stransky, N. et al. The mutational landscape of head and neck squamous cell carcinoma. Science 333, 1157-1160 (2011)
5. Lleras, R. A. et al. Unique DNA methylation loci distinguish anatomic site and HPV status in head and neck squamous cell carcinoma. Clin. Cancer Res.: Off. J. Am. Assoc. Cancer Res. 19, 5444-5455 (2013).

6. Guerrero-Preston, R. et al. Key tumor suppressor genes inactivated by "greater promoter" methylation and somatic mutations in head and neck cancer. Epigenetics: Off. J. DNA Methyl. Soc. 9, 1031-1046 (2014).

7. Chung, C. H. et al. Molecular classification of head and neck squamous cell carcinomas using patterns of gene expression. Cancer Cell 5, 489-500 (2004).

8. Cancer Genome Atlas, N.. Comprehensive genomic characterization of head and neck squamous cell carcinomas. Nature 517, 576-582 (2015).

9. Argiris, A. et al. Prognostic significance of human papillomavirus in recurrent or metastatic head and neck cancer: an analysis of eastern cooperative oncology Group trials. Ann. Oncol.: Off. J. Eur. Soc. Med. Oncol. 25, 1410-1416 (2014).

10. Mroz, E. A., Tward, A. D., Hammon, R. J., Ren, Y. \& Rocco, J. W. Intra-tumor genetic heterogeneity and mortality in head and neck cancer: analysis of data from the cancer genome atlas. PLoS Med. 12, e1001786 (2015).

11. Mo, Q. et al. Pattern discovery and cancer gene identification in integrated cancer genomic data. Proc. Natl Acad. Sci. USA 110, 4245-4250 (2013).

12. Gross, A. M. et al. Multi-tiered genomic analysis of head and neck cancer ties TP53 mutation to 3p loss. Nat. Genet. 46, 939-943 (2014).

13. Lek, M. et al. Analysis of protein-coding genetic variation in 60,706 humans. Nature 536, 285-291 (2016).

14. Gregory, P. A. et al. The miR-200 family and miR-205 regulate epithelial to mesenchymal transition by targeting ZEB1 and SIP1. Nat. Cell. Biol. 10, 593-601 (2008).

15. Byers, L. A. et al. An epithelial-mesenchymal transition gene signature predicts resistance to EGFR and PI3K inhibitors and identifies Axl as a therapeutic target for overcoming EGFR inhibitor resistance. Clin. Cancer Res.: Off. J. Am. Assoc. Cancer Res. 19, 279-290 (2013).

16. Subramanian, A. et al. Gene set enrichment analysis: a knowledge-based approach for interpreting genome-wide expression profiles. Proc. Natl Acad. Sci. USA 102, 15545-15550 (2005).

17. Love, M. I., Huber, W. \& Anders, S. Moderated estimation of fold change and dispersion for RNA-seq data with DESeq2. Genome Biol. 15, 550 (2014).

18. Kurotaki, N. et al. Haploinsufficiency of NSD1 causes Sotos syndrome. Nat. Genet. 30, 365-366 (2002).

19. Morishita, M. \& di Luccio, E. Cancers and the NSD family of histone lysine methyltransferases. Biochim. Biophys. Acta 1816, 158-163 (2011).

20. Jaffe, J. D. et al. Global chromatin profiling reveals NSD2 mutations in pediatric acute lymphoblastic leukemia. Nat. Genet. 45, 1386-1391 (2013).

21. $\mathrm{Lu}, \mathrm{T}$. et al. Regulation of NF-kappaB by NSD1/FBXL11-dependent reversible lysine methylation of p65. Proc. Natl Acad. Sci. USA 107, 46-51 (2010)

22. Nakshatri, H. et al. NF-kappaB-dependent and -independent epigenetic modulation using the novel anti-cancer agent DMAPT. Cell Death Dis. 6, e1608 (2015).

23. Lee, S. T. \& Wiemels, J. L. Genome-wide CpG island methylation and intergenic demethylation propensities vary among different tumor sites. Nucleic Acids Res. 44, 1105-1117 (2016).

24. Choufani, S. et al. NSD1 mutations generate a genome-wide DNA methylation signature. Nat. Commun. 6, 10207 (2015).

25. Baubec, T. et al. Genomic profiling of DNA methyltransferases reveals a role for DNMT3B in genic methylation. Nature 520, 243-247 (2015).

26. Cedar, H. \& Bergman, Y. Linking DNA methylation and histone modification: patterns and paradigms. Nat. Rev. Genet. 10, 295-304 (2009).

27. Li, H. et al. The histone methyltransferase SETDB1 and the DNA methyltransferase DNMT3A interact directly and localize to promoters silenced in cancer cells. J. Biol. Chem. 281, 19489-19500 (2006).

28. Neri, F. et al. Intragenic DNA methylation prevents spurious transcription initiation. Nature 543, 72-77 (2017).

29. Papillon-Cavanagh, S. et al. Impaired H3K36 methylation defines a subset of head and neck squamous cell carcinomas. Nat. Genet. 49, 180-185 (2017).

30. Berdasco, M. et al. Epigenetic inactivation of the Sotos overgrowth syndrome gene histone methyltransferase NSD1 in human neuroblastoma and glioma. Proc. Natl Acad. Sci. USA 106, 21830-21835 (2009).

31. Wang, G. G., Cai, L., Pasillas, M. P. \& Kamps, M. P. NUP98-NSD1 links H3K36 methylation to Hox-A gene activation and leukaemogenesis. Nat. Cell. Biol. 9 , 804-812 (2007).

32. Soukup, V., Horacek, I. \& Cerny, R. Development and evolution of the vertebrate primary mouth. J. Anat. 222, 79-99 (2013).

33. Sato, T., Issa, J. J. \& Kropf, P. DNA Hypomethylating drugs in cancer therapy. Cold Spring Harbor Perspect. Med. 7, doi:10.1101/cshperspect.a026948 (2017).

34. Kim, K. H. \& Roberts, C. W. Targeting EZH2 in cancer. Nat. Med. 22, 128-134 (2016).

35. Yamagishi, M. \& Uchimaru, K. Targeting EZH2 in cancer therapy. Curr. Opin. Oncol. 29, 375-381 (2017).

36. Popovic, R. et al. Histone methyltransferase MMSET/NSD2 alters EZH2 binding and reprograms the myeloma epigenome through global and focal changes in H3K36 and H3K27 methylation. PLoS Genet. 10, e1004566 (2014). 
37. Chin, L., Hahn, W. C., Getz, G. \& Meyerson, M. Making sense of cancer genomic data. Genes Dev. 25, 534-555 (2011).

38. van de Wiel, M. A., Picard, F., van Wieringen, W. N. \& Ylstra, B. Preprocessing and downstream analysis of microarray DNA copy number profiles. Brief Bioinform. 12, 10-21 (2011).

39. Reva, B., Antipin, Y. \& Sander, C. Predicting the functional impact of protein mutations: application to cancer genomics. Nucleic Acids Res. 39, e118 (2011).

40. Gao, J. et al. Integrative analysis of complex cancer genomics and clinical profiles using the cBioPortal. Sci. Signal. 6, pl1 (2013).

41. Du, P. et al. Comparison of Beta-value and M-value methods for quantifying methylation levels by microarray analysis. BMC Bioinf. 11, 587 (2010).

42. Laddha, S. V., Ganesan, S., Chan, C. S. \& White, E. Mutational landscape of the essential autophagy gene BECN1 in human cancers. Mol. Cancer Res. 12, 485-490 (2014)

43. Pidsley, R. et al. A data-driven approach to preprocessing Illumina $450 \mathrm{~K}$ methylation array data. BMC Genomics 14, 293 (2013).

44. Benjamini, Y. \& Hochhberg, Y. Controlling the false discovery rate: a practical and powerful approach to multiple testing. J. R. Stat. Soc. B 57, 289-300 (1995).

45. Law, C. W., Chen, Y., Shi, W. \& Smyth, G. K. voom: precision weights unlock linear model analysis tools for RNA-seq read counts. Genome Biol. 15, R29 (2014).

46. Barbie, D. A. et al. Systematic RNA interference reveals that oncogenic KRASdriven cancers require TBK1. Nature 462, 108-112 (2009).

47. Huang da, W., Sherman, B. T. \& Lempicki, R. A. Systematic and integrative analysis of large gene lists using DAVID bioinformatics resources. Nat. Protoc. 4, 44-57 (2009).

48. Streit, M. et al. Guided visual exploration of genomic stratifications in cancer. Nat. Methods 11, 884-885 (2014).

49. Hoque, M. O. et al. Quantitative methylation-specific polymerase chain reaction gene patterns in urine sediment distinguish prostate cancer patients from control subjects. J. Clin. Oncol.: Off. J. Am. Soc. Clin. Oncol. 23, 6569-6575 (2005).

50. Li, H. \& Durbin, R. Fast and accurate short read alignment with BurrowsWheeler transform. Bioinformatics 25, 1754-1760 (2009).

51. DePristo, M. A. et al. A framework for variation discovery and genotyping using next-generation DNA sequencing data. Nat. Genet. 43, 491-498 (2011).

52. Cibulskis, K. et al. Sensitive detection of somatic point mutations in impure and heterogeneous cancer samples. Nat. Biotechnol. 31, 213-219 (2013).

53. Spencer, D. H. et al. Performance of common analysis methods for detecting low-frequency single nucleotide variants in targeted next-generation sequence data. J. Mol. Diagnos. 16, 75-88 (2014).

54. Wang, K., Li, M. \& Hakonarson, H. ANNOVAR: functional annotation of genetic variants from high-throughput sequencing data. Nucleic Acids Res. 38, e164 (2010).

55. Koboldt, D. C. et al. VarScan: variant detection in massively parallel sequencing of individual and pooled samples. Bioinformatics 25, 2283-2285 (2009).

56. Vaser, R., Adusumalli, S., Leng, S. N., Sikic, M. \& Ng, P. C. SIFT missense predictions for genomes. Nat. Protoc. 11, 1-9 (2016).

57. Adzhubei, I., Jordan, D. M. \& Sunyaev, S. R. Predicting functional effect of human missense mutations using PolyPhen-2. In Current protocols in human genetics/editorial board(Jonathan L. Haines et al.) Chapter 7, Unit7 20, doi:10.1002/0471142905.hg0720s76 (2013).

58. Anisimova, M., Bielawski, J. P. \& Yang, Z. Accuracy and power of the likelihood ratio test in detecting adaptive molecular evolution. Mol. Biol. Evol. 18, 1585-1592 (2001).
59. Shihab, H. A. et al. Predicting the functional, molecular, and phenotypic consequences of amino acid substitutions using hidden markov models. Hum. Mutat. 34, 57-65 (2013).

60. Therneau, T. M. \& Grambsch, P. M. Modeling Survival Data: Extending the Cox Model (Springer-Verlag, 2010).

\section{Acknowledgements}

We were supported by NCI Core Grant P30 CA006927 (to Fox Chase Cancer Center) NCI CA181287, and the Don Devlin Fund (to E.A.G.), pilot Genomics Funding from Temple University and support from the Beck family (to S.P. and E.A.G.), Swiss Cancer League BIL KLS-3649-02-2015 (to A.D.S.), and NIH grant SPORE P50 DE019032 (to D. S.). We thank Anna Nikonova, Emmanuelle Nicholas, and members of Biosample Repository Facility of Fox Chase Cancer Center for their extensive support.

\section{Author contributions}

S.P. and E.A.G. conceived the study; S.P. and M.J.S. performed most analyses; S.P. and K.R. performed survival analyses; I.G.S. aided in the bioinformatics analyses; E.I. and A.D.S designed experiments and generated sequence data for validation study; E.I., A.D.S. and T.G. compiled clinical follow-up data for JH validation cohort; B.A.B., R.M. and D.S. provided clinical expertise; D.S. supported JH validation; E.R. provided statistical and bioinformatics expertise; and S.P. and E.A.G. wrote the manuscript with assistance from the coauthors.

\section{Additional information}

Supplementary Information accompanies this paper at doi:10.1038/s41467-017-01877-7.

Competing interests: The authors declare no competing financial interests.

Reprints and permission information is available online at http://npg.nature.com/ reprintsandpermissions/

Publisher's note: Springer Nature remains neutral with regard to jurisdictional claims in published maps and institutional affiliations.

(c) (i) Open Access This article is licensed under a Creative Commons Attribution 4.0 International License, which permits use, sharing, adaptation, distribution and reproduction in any medium or format, as long as you give appropriate credit to the original author(s) and the source, provide a link to the Creative Commons license, and indicate if changes were made. The images or other third party material in this article are included in the article's Creative Commonslicense, unless indicated otherwise in a credit line to the material. If material is not included in the article'sCreative Commons license and your intended use is not permitted by statutory regulation or exceeds the permitted use, you will need to obtain permission directly from the copyright holder. To view a copy of this license, visit http://creativecommons.org/ licenses/by/4.0/.

(C) The Author(s) 2017 